\title{
Errors and limitations of the multimodality checking methods of defective spinal intrathecal pump systems. Case report
}

\author{
B Schurch MD
}

Swiss Paraplegic Centre Balgrist, CH 8008 Zurich, Switzerland.

The loss of benefit from intrathecal baclofen, with increased spasticity and a discrepancy between the residual and the calculated volume content (underinfusion), made us suspect dysfunction of the intrathecal baclofen infusion in a paraplegic patient. Although all possible usual checking methods were used, no failure in the drug administration device (DAD) could be found. Despite some benefit from increasing the daily dose of baclofen, it has not been possible to control the spastic symptoms. Surgery was therefore decided upon, and a small leak at the end of the catheter tip was discovered. Surgeons and physicians should be aware that checking methods of DAD cannot exclude failure of the system. Their errors and limitations are discussed.

Keywords: baclofen; spasticity; intrathecal drug infusion; spinal cord disease; infusion pump.

\section{Introduction}

Intrathecal drug administration devices (DAD) are being increasingly used in the treatment of intractable pain, rigidity and spasm in spinal cord lesioned patients.

Baclofen has been shown to be efficient for the treatment of rigidity and spasms, when administrated intrathecally. ${ }^{1}$ Various problems can nevertheless occur during such treatment: tachyphylaxy (tolerance), overdose or underdose following programming errors or technical failure of the pump system. $^{2-7}$

In cases of suspected pump failures, physicians should, after having eliminated any other possible cause of the increased spasticity, completely check the DAD according to an established protocol. ${ }^{8}$

We report a patient with suspected DAD dysfunction, where the diagnosis of failure could not be made according to this protocol, thus making surgery inevitable.

\section{Case report}

A 24 year old man was involved in an accident in April 1987 with a consequent fracture-dislocation T8 and complete spastic paraplegia below $\mathrm{T} 8$.

In April 1991, due to intractable spasm, implantation of the drug administration device
(DAD) was performed. A 4F 'Silastic catheter' ((Model 8703) Metronic Inc, Minneapolis USA) was introduced through a Tuohy needle, under general anaesthesia between L3 and L4 and the tip pushed rostrally $10-15 \mathrm{~cm}$ into the subarachnoid space, reaching, at least, the T11-L1 level. The catheter position was confirmed intraoperatively by fluoroscopy and postoperatively by radiography. The catheter was sutured to the fascia and tunnelled subcutaneously to the left abdominal wall, where a pocket for the DAD was made. The DAD was also sutured to the fascia and was connected to the catheter. The implantable pump (Synchromed, Model $8611 \mathrm{H}$, Metronic Inc, Minneapolis USA) was filled with $18 \mathrm{ml}$ of the drug in a concentration of $500 \mu \mathrm{g} / \mathrm{ml}$ (delivered by Ciba Geigy Corp, Basel, Switzerland). The flow was started shortly after implantation, in a continuous infusion mode. Drug delivery was programmed through a physician's microcomputer and transmitted to the implanted pump noninvasively via a hand held radiofrequency telemetry wand. A daily dose of $60 \mu \mathrm{g}$ was found to be adequate to control the spasticity. Refills were performed by a percutaneous puncture through a rubber septum in the pump device with intervals of 90 days.

In December 1991 the patient presented because of recent increase of spasms. No particularity returned to the anamnesis. Neurostatus testing of rigidity and spasms showed an Ashworth scale score of 5 and 5 , respectively. No causes of increased nociceptive response could 
be found and general laboratory tests remained without any specificity. Checking of the pump function showed a discrepancy between residual volume content and calculated volume content (underinfusion). Further investigations revealed no reason for DAD dysfunction. Neither catheter disconnection, kink or dislodgement were seen in the plain xray (Fig 1). Control of the pump-rotor movement, by programming a bolus calculated as described to produce a rotor movement of $90^{\circ}$ in $45 \mathrm{~s}$.

$$
\frac{1800}{120 \text { (cal const) }}=15 \mu \mathrm{l}=7.4 \mu \mathrm{g}
$$

implied an adequate pump function (Fig 2).

Filling up the pump with $10 \mathrm{ml}$ of a radioopaque solution (Iopamiro) did not provide any useful information. This solution could not be detected, either at the catheter tip, or at a possible site of catheter dysfunction, even after three boluses of $0.5 \mathrm{ml}$ (Fig 3).

Filling up the pump with a radiolabelled solution $(1.5 \mathrm{ml}$ in $10 \mathrm{ml} \mathrm{NaCl}, 30 \mathrm{Mbq}$ IN 111 DTPA) with consecutive bolus and delivering the solution during 24 hours revealed normal pump filling without any abnormal activity

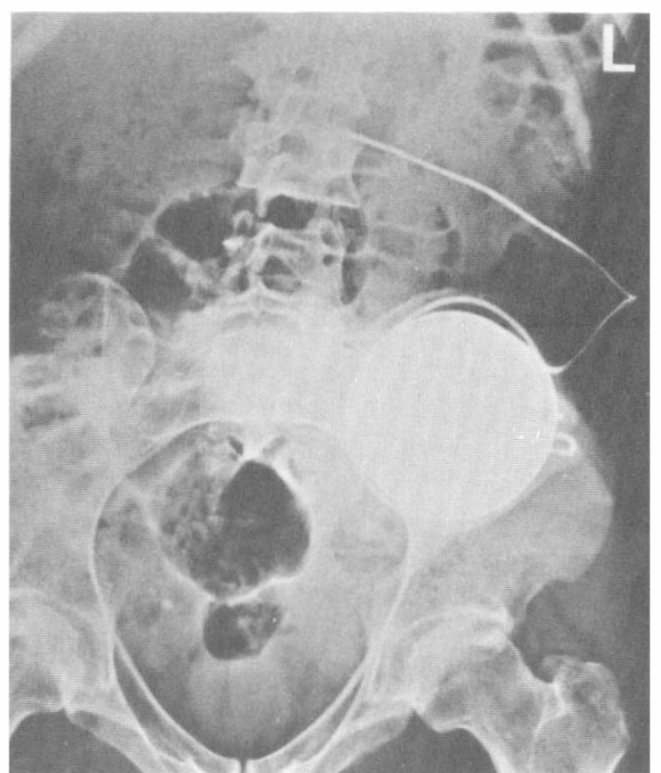

Figure 1 Plain xrays check. Neither catheter disconnection, nor kink, nor dislodgement are seen on the plain xrays.

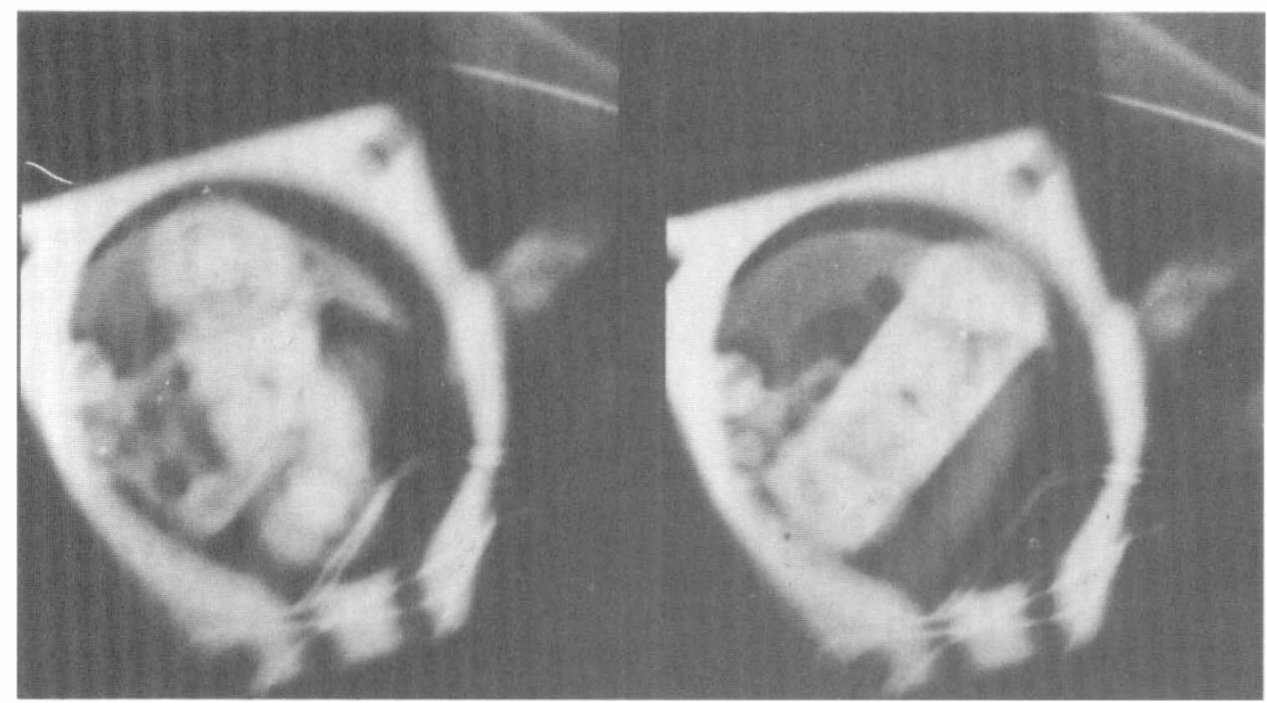

Figure 2 Check of pump rotor movement by programming a bolus calculated as described to produce a rotor movement of $90^{\circ}$ in $45 \mathrm{~s}$.

$$
\frac{1800}{120 \text { (cal const })}=15 \mu \mathrm{l}=7.5 \mu \mathrm{g}
$$

seemed to show an adequate pump function. 


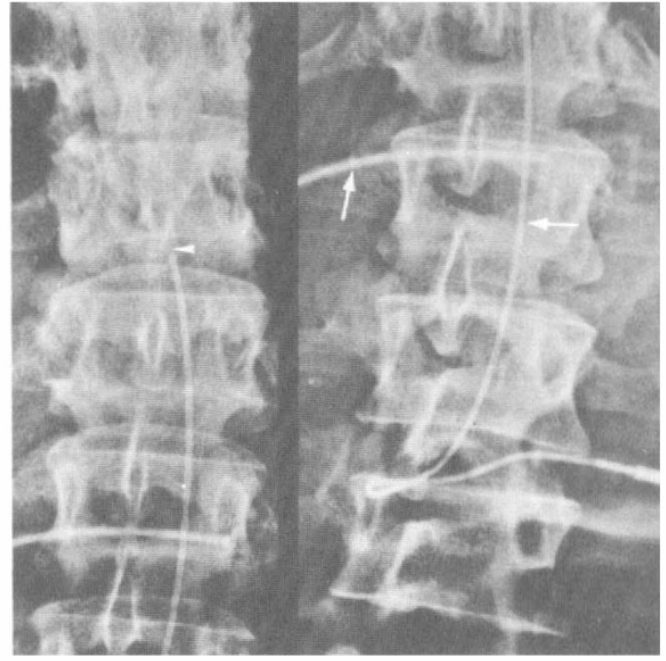

Figure 3 Filling the pump with $10 \mathrm{ml}$ radioopaque solution and bolus three times. Radiologic solution could not be detected, either at the catheter tip, or at a possible site of catheter disconnection, even after three boluses of $0.5 \mathrm{ml}$. Furthermore, because of the usual radioopacity of the implanted catheter (Fig 1), it was not possible to arrive at any conclusion regarding the perfusion of the solution (as shown when compared with a similar outside catheter filled with the radioopaque solution (white arrows)).

around the pump. No change was seen after 6 hours. Serial scans after 24 hours showed diffusion of the material at the end of the catheter, throughout the cerebrospinal fluid (CSF) but nowhere else, suggesting that the catheter had been positioned intrathecally and had no leak (Fig 4).

After these investigations, DAD dysfunction was excluded and the daily dose of baclofen was increased. Despite slight improvement it had not been possible to reduce the spasticity, thus making surgical intervention inevitable. A very small disconnection at the distal part of the catheter was discovered.

\section{Discussion}

Direct spinal intrathecal administration of specific gamma aminobutyric acid (GABA) -B-agonist (baclofen) is a relatively new pharmacological concept in the management of spasticity by spinal cord lesion patients. This method, proposed by Penn

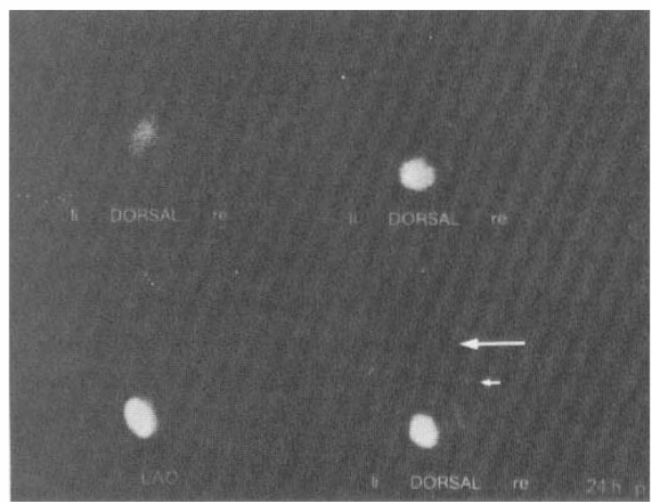

Figure 4 Filling the pump with $10 \mathrm{ml}$ radiolabelled indium solution, bolus, and delivering the solution during 24 hours. Serial scans after 24 hours showed diffusion of the material at the end of the catheter (small arrow), throughout the CSF (big arrow) but nowhere else, thereby suggesting that the catheter has been positioned intrathecally and has no leak.

and Kroin ${ }^{1}$ who conducted further experimental work on this approach, ${ }^{9,10}$ has been widely used and reported on by many authors. ${ }^{11-14}$ Long term follow ups are now available. ${ }^{2,3,15}$ The efficiency and safety of this method can be reached only on the condition that the selection and prescription criteria are rigorously respected. However, various problems can occur during such treatment: tachyphylaxy (tolerance), overdose or underdose following programming errors or technical failure of the system have in particular been described. ${ }^{2-7}$ In cases of suspected pump dysfunction it is proposed, after eliminating any other cause of increased spasticity, to check the DAD according to a recommended protocol (Fig 5).

From our experience it appears, however, that:

1 Xray projection errors cannot provide an accurate evaluation of the pump function by programming the recommended $90^{\circ}$ rotor movement. $360^{\circ}$ rotor movement should be preferred, combined with a control under fluoroscopy or an xray at the middle of the programmed time to be sure that the pump is running.

2 Filling up the pump with $5-10 \mathrm{ml}$ of a 


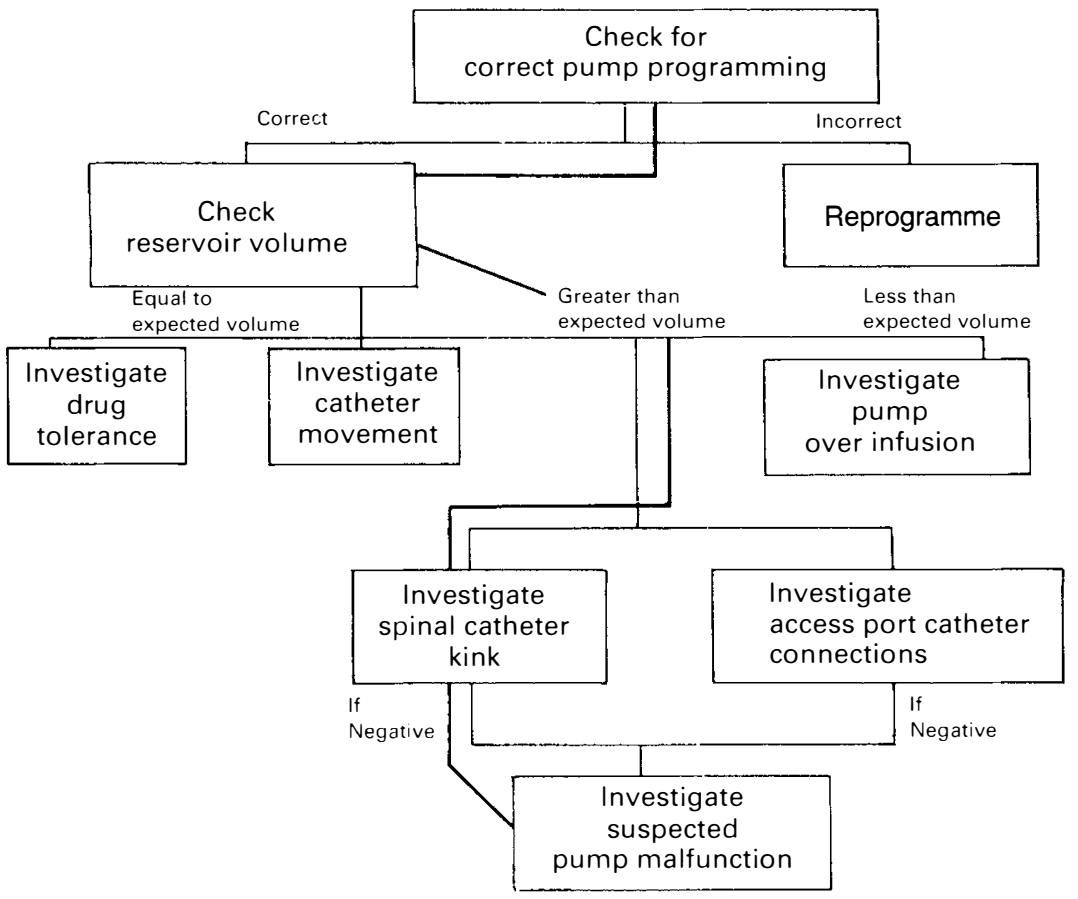

- Procedure to check DAD

The way we checked DAD

Figure 5 General protocol recommended for checking DAD dysfunction.

radioopaque solution and injecting a bolus of it cannot give information on the presence of solution inside the catheter. Furthermore, it seems impossible to detect the radioopaque solution at the tip of the catheter, as a minimum of $10 \mathrm{ml}$ is usually necessary to perform a myelogram, considering the dilution ratio. CT scan should be able to detect the solution at the catheter tip. ${ }^{16}$

3 Filling up the pump with a radiolabelled solution with consecutive serial scans after 24 hours $^{8,16}$ cannot, with a high degree of accuracy, exclude a small leakage around the catheter system. Serial scans after 6 hours appear to us useless if there is a suspicion of underinfusion.
It remains unclear if an additional external compression of the catheter in a very thin patient could be responsible for an underinfusion of the system, resulting in a discrepancy between the measured residual volume content and the calculated volume content, as observed in our patient.

Surgeons and physicians should be aware of the possible diagnostic errors and limitations of multimodality checking methods of defective intrathecal pump systems. According to us, surgical revision should be performed in cases of negative evaluation of a pump (or catheter system) dysfunction, when no improvement of the symptomatology occurs after increasing the daily dose of baclofen.

\section{References}

1 Penn RD, Kroin JS (1984) Intrathecal baclofen alleviates spinal cord spasticity. Lancet 1: 1078 (letter).

2 Lazorthes Y, Sallerin-Caute B, Verdie JC, Bastide R, Carillo JP (1990) Chronic intrathecal baclofen administration for control of severe spasticity. J Neurosurg 72(3): 393-402.

3 Penn RD (1992) Intrathecal baclofen for spasticity of spinal origin: seven years of experience. J Neurosurg 77(2): $236-40$. 
4 Loubser PG, Narayan RK, Sandin KJ, Donovan WIH, Russell KD (1991) Continuous infusion of intrathecal baclofen: Long term effects on spasticity in spinal cord injury. Paraplegia 29(1): 48-64.

5 Eddy P, Jamous A, Gardner B, Wang D, Silver J (1992) Complications of intrathecal drug delivery. Br J Neurosurg 6(2): 115-8.

6 Delhaas EM, Brouwers JR (1991) Intrathecal overdose: Report of 7 events in 5 patients and review of the literature. Int J Clin Pharmacol Ther Toxicol 29(7): 274-80.

7 Zierski J, Muller H, Dralle D, Wurdinger T (1988) Implanted pump systems for treatment of spasticity. Acta Neurochir Suppl (Wien) 43: 94-9.

8 Medtronic (1987): Users' Guide to Medtronic Synchromed Infusionsystem. Medtronic Inc, Minneapolis.

9 Kroin JS, Penn RD, Beissinger RL, Arzbaecher RC (1984) Reduced spinal reflexes following intrathecal baclofen in the rabbit. Exp Brain Res 54: 191-19.

10 Penn RD, Kroin JS (1985) Continuous intrathecal baclofen for severe spasticity. Lancet 2: 125-127.

11 Hankey GJ, Stewart-Wynne EG, Perlman D (1986) Intrathecal baclofen for severe spasticity. Med J Aust 145: $465-466$.

12 Lazorthes Y (1987) Chronic intrathecal administration of baclofen in treatment of severe spasticity. In: Müller H, Zierski J, Penn RD, editors. Local Spinal Therapy of Spasticity. Springer-Verlag, Berlin: 215-222.

13 Siegfried J, Rea GL (1987) Intrathecal application of baclofen in treatment of spasticity. Acta Neurochir Suppl 39: 121-123.

14 Penn RD, Kroin JS (1987) Long term intrathecal baclofen infusion for treatment of spasticity. J Neurosurg 66(2): $181-5$.

15 Ochs G, Struppler A, Meyerson BA, Linderoth B, Gybels J, Gardner BP et al. (1989). Intrathecal baclofen for long-term treatment of spasticity. A multicenter-study. J Neurol Neurosurg Psychiatry 52(8): 933-9.

16 Ochs G, Delhaas EM (1992) Spasticity management with the Synchromed ${ }^{\mathrm{TM}}$ Infusion System. In: The Synchromed ${ }^{T M}$ Infusion System. Medtronic Inc (Schweiz), Dübendorf. 